\title{
BMJ Open Association of prehypertension and hyperhomocysteinemia with subclinical atherosclerosis in asymptomatic Chinese: a cross-sectional study
}

\author{
Bo Liu, ${ }^{1}$ Zhihao Chen, ${ }^{2}$ Xiaoqi Dong, ${ }^{3}$ Guangming Qin ${ }^{1}$
}

To cite: Liu B, Chen Z, Dong $\mathrm{X}$, et al. Association of prehypertension and hyperhomocysteinemia with subclinical atherosclerosis in asymptomatic Chinese: a crosssectional study. BMJ Open 2018;8:e019829. doi:10.1136/ bmjopen-2017-019829

- Prepublication history for this paper is available online. To view these files, please visit the journal online (http://dx.doi org/10.1136/bmjopen-2017019829).

$\mathrm{BL}$ and $\mathrm{ZC}$ contributed equally.

Received 6 0ctober 2017 Revised 25 January 2018 Accepted 29 January 2018

\section{Check for updates}

${ }^{1}$ Department of Laboratory, School of Medicine, Second Affiliated Hospital, Zhejiang University, Hangzhou, China ${ }^{2}$ Department of Infectious Diseases, School of Medicine, Second Affiliated Hospital, Zhejiang University, Hangzhou, China

${ }^{3}$ Department of Oral Maxillofacial Surgery, School of Medicine, Second Affiliated Hospital, Zhejiang University, Hangzhou, China

Correspondence to

Guangming Qin;

zejykqgm@zju.edu.cn

\section{ABSTRACT}

Objectives Comorbid hypertension and

hyperhomocysteinemia is an important risk factor for carotid atherosclerotic plaque formation. We put forward the hypothesis that the subjects with comorbid prehypertension and hyperhomocysteinemia also had an increased risk of subclinical atherosclerosis, using carotid intima-media thickness (CIMT) as the marker of the atherosclerotic process.

Methods A total of 4102 asymptomatic Chinese subjects aged 18-60 years were divided into four groups according to blood pressure (BP) and homocysteine (HCY) level: the control group without prehypertension or hyperhomocysteinemia, isolated prehypertension group, simple hyperhomocysteinemia group and prehypertension with hyperhomocysteinemia group. Serum lipids, fasting blood glucose (FBG), HCY and CIMT were measured.

Results There was significant difference in the positive rates of increased CIMT among four groups. Compared with the controls, the subjects in the other three groups had a higher risk of increased CIMT (isolated prehypertension group, OR $2.049,95 \% \mathrm{Cl}$ 1.525 to 2.754 ; simple hyperhomocysteinemia group, OR $2.145,95 \% \mathrm{Cl} 1.472$ to 3.125 ; prehypertension and hyperhomocysteinemia group, $0 \mathrm{R} 3.199,95 \% \mathrm{Cl} 2.362$ to 4.332). However, by multiple logistic regression analysis, only comorbid prehypertension and hyperhomocysteinemia was independently associated with increased CIMT (OR $1.485,95 \% \mathrm{Cl} 1.047$ to $2.108, \mathrm{P}<0.05)$.

Conclusions Comorbid prehypertension and hyperhomocysteinemia was an independent risk factor of subclinical atherosclerosis in asymptomatic Chinese, but isolated prehypertension or hyperhomocysteinemia was not. Therefore, combined intervention for prehypertension and hyperhomocysteinemia may contribute to decrease the incident of cardiovascular disease.

\section{INTRODUCTION}

Atherosclerosis is a complex chronic disease related with a variety of risk factors, which play an important role in the generation of cardiovascular disease. ${ }^{1}$ Many studies have identified the risk factors of atherosclerosis, including smoking, adiposity, blood pressure (BP), blood cholesterol, diabetes mellitus

\section{Strengths and limitations of this study}

This is the first study to focus on the association of comorbid prehypertension and hyperhomocysteinemia with subclinical atherosclerosis in China.

- Many confounders including traditional risk factors, metabolism confounders, demographic factors and lifestyle habits have been taken into consideration and adjusted to control bias.

- Some definitions (hyperhomocysteinemia and increased carotid intima-media thickness) were according to Chinese guidelines which might be different from other countries.

- Because of the cross-sectional study design, the speculation regarding causality is limited and requires further research.

and hyperhomocysteinemia. ${ }^{2}$ Preventive interventions according to these risk factors in subclinical stage contribute to the declines in incident cardiovascular events. ${ }^{3}$ Carotid intima-media thickness (CIMT) detected by ultrasonography is a reliable marker of subclinical atherosclerosis. ${ }^{4}$

Many studies were conducted to investigate the association between hyperhomocysteinemia and subclinical atherosclerosis in general population. Several studies suggested hyperhomocysteinemia was not correlated with subclinical atherosclerosis. ${ }^{5-7}$ A cohort of 1111 subjects survey showed inconsistent results which revealed that mild hyperhomocysteinemia was an independent risk factor for increased carotid artery wall thickness. ${ }^{8}$ However, it has been well established that elevated homocysteine (HCY) level was involved in progression of subclinical atherosclerosis in the patients with hypertension. ${ }^{9-11}$ Recent studies also suggested that comorbid hypertension and hyperhomocysteinemia showed combined effect on the generation of subclinical atherosclerosis in general population. ${ }^{12} 13$ 


\begin{tabular}{|c|c|c|c|}
\hline Characteristics & $\begin{array}{l}\text { Normal } \\
\text { CIMT }(<0.9 \mathrm{~mm})\end{array}$ & $\begin{array}{l}\text { Increased } \\
\text { CIMT ( } \geq 0.9 \mathrm{~mm})\end{array}$ & $\mathbf{P}$ \\
\hline Age (Year) & $43 \pm 8$ & $51 \pm 6$ & $<0.001$ \\
\hline $\mathrm{SBP}(\mathrm{mm} \mathrm{Hg})$ & $121 \pm 10$ & $124 \pm 9$ & $<0.001$ \\
\hline $\mathrm{DBP}(\mathrm{mm} \mathrm{Hg})$ & $74 \pm 8$ & $76 \pm 8$ & $<0.001$ \\
\hline $\mathrm{BMI}\left(\mathrm{kg} / \mathrm{m}^{2}\right)$ & $23.7 \pm 3.0$ & $24.7 \pm 2.7$ & $<0.001$ \\
\hline $\mathrm{CHO}(\mathrm{mmol} / \mathrm{L})$ & $4.94 \pm 0.93$ & $5.30 \pm 0.98$ & $<0.001$ \\
\hline $\mathrm{HDL}(\mathrm{mmol} / \mathrm{L})$ & $1.46 \pm 0.34$ & $1.40 \pm 0.33$ & 0.003 \\
\hline LDL (mmol/L) & $3.06 \pm 0.78$ & $3.43 \pm 0.80$ & $<0.001$ \\
\hline $\mathrm{TG}(\mathrm{mmol} / \mathrm{L})$ & $1.68 \pm 1.11$ & $1.88 \pm 0.97$ & 0.001 \\
\hline $\mathrm{FBG}(\mathrm{mmol} / \mathrm{L})$ & $5.30 \pm 0.99$ & $5.68 \pm 1.35$ & $<0.001$ \\
\hline $\mathrm{HCY}(\mu \mathrm{mol} / \mathrm{L})$ & $9.62 \pm 4.58$ & $10.56 \pm 4.38$ & $<0.001$ \\
\hline Gender & & & $<0.001$ \\
\hline Male & 2137 & 292 & \\
\hline Female & 1557 & 116 & \\
\hline Smoking status & & & $<0.001$ \\
\hline Non-smoker & 2784 & 267 & \\
\hline Smoker & 910 & 141 & \\
\hline
\end{tabular}

Continued variables were expressed as mean \pm SD and compared using Student's t-test; the categorical variables were expressed as number and analysed by $\chi^{2}$ test.

$\mathrm{BMI}$, body mass index; $\mathrm{CHO}$, total cholesterol; DBP, diastolic blood pressure; FBG, fasting blood glucose; HCY, homocysteine; HDL, high-density lipoprotein; LDL, low-density lipoprotein; SBP, systolic blood pressure; TG, triglycerides.

Prehypertension is prevalent in asymptomatic population. A survey based on 17584 subjects in China suggested that the prevalence of prehypertension was about $36.0 \%$ in general population. ${ }^{14}$ It is meant that prehypertension has not progressed to hypertension in many general populations. However, few studies devoted to the probable association of comorbid prehypertension and hyperhomocysteinemia with subclinical atherosclerosis. Therefore, we investigated this association in asymptomatic Chinese, using CIMT as the marker of the atherosclerotic process.

\section{METHODS}

\section{Subjects}

The survey used a random sample of 5822 subjects. All participants were admitted to the Second Affiliated Hospital of Zhejiang University School of Medicine to have a general health examination from September 2014 to December 2015. All the subjects were asked to complete a questionnaire by similar investigators trained in inquiry methods, including gender, date of birth, occupation, marital status, smoking status, alcohol intake history, medical history and family history. After exclusion of participants with cardiovascular and cerebrovascular disease, atherosclerotic disease, cancer, hypotension, hypertension, diabetes, renal disease, hepatic disease, thyroid disease, infectious diseases, heavy drinkers ${ }^{15}$ or any drug therapy, the final sample size was 4102 (2429 men and 1673 women: aged 18-60).

The subjects were divided into four groups according to BP and HCY level: the control group without prehypertension or hyperhomocysteinemia, isolated prehypertension group, simple hyperhomocysteinemia group and prehypertension with hyperhomocysteinemia group. An HCY level $\geq 10 \mu \mathrm{mol} / \mathrm{L}$ was defined as hyperhomocysteinemia. ${ }^{16}$ Occasional or smoke $\geq 1 \mathrm{cig} /$ day was defined as smoker. ${ }^{17}$ Prehypertension was defined as BP between 120 and $139 \mathrm{~mm} \mathrm{Hg}$ systolic or 80 and $89 \mathrm{~mm} \mathrm{Hg}$ diastolic. ${ }^{18}$ Normotension was defined as BP values $<120 / 80 \mathrm{~mm} \mathrm{Hg}$.

\section{Physical examination and laboratory methods}

The body mass index (BMI) was calculated as the weight $(\mathrm{kg})$ divided by the square of the height $\left(\mathrm{m}^{2}\right)$. The BP was measured in right arm, using an automated device (Omron 711, USA), with the patient in a seated position. The participant had refrained from caffeinated beverages, smoking and exercise for $30 \mathrm{~min}$ and had rested quietly for at least $10 \mathrm{~min}$. The means of twice BP measurements ( 5 min between each) were recorded. In cases of a difference between the two measurements greater than $10 \mathrm{~mm} \mathrm{Hg}$, a third measurement followed.

All the subjects fasted overnight (at least 12 hours). All the blood samples were collected into $5 \mathrm{~mL}$ gel separator tubes (BD, USA) between $8 \mathrm{AM}$ and $10 \mathrm{AM}$. The samples were centrifuged at 3000 revolutions/min immediately. Serum was subsequently isolated from the whole blood. All of the analyses including fasting blood glucose (FBG), total cholesterol (CHO), high-density lipoprotein cholesterol (HDL-C), low-density lipoprotein cholesterol (LDL$\mathrm{C})$, triglycerides (TG) and HCY were performed with a Beckman AU5800 automatic analyser (Beckman Coulter, Tokyo, Japan) within 1 hour. The HCY was measured by an enzymatic cycling method. Axis-Shield HCY Control Kit was used as the daily quality control (intra-assay coefficient of variation $<5 \%$, interassay coefficient of variation $<6 \%$ ).

\section{Carotid ultrasound measurement}

The distal segment, stigma compartments of the cephalic artery and the proximal segment of the internal carotid artery were measured on both sides. ${ }^{19}$ Each blood vessel was measured in three sections within a range of $1 \mathrm{~cm}$ in the proximal wall and distant from the side walls. The subject was defined with increased CIMT when the CIMT $\geq 0.9 \mathrm{~mm}{ }^{20}$

\section{Statistics}

The statistical analysis was performed using the IBM SPSS V.20.0 software package. Continued variables were expressed as the mean $\pm \mathrm{SD}$ and compared using Student's t-test. The categorical variables were analysed by $\chi^{2}$ test. The OR and $95 \% \mathrm{CI}$ were calculated by logistic regression analysis. Multivariate logistic regression 
Table 2 The ORs for increased carotid intima-media thickness (CIMT) in different blood pressure and homocysteine (HCY) groups

\begin{tabular}{|c|c|c|c|c|c|c|c|}
\hline Groups & $\begin{array}{l}\text { CIMT } \\
\text { positive (N) }\end{array}$ & OR $(95 \% \mathrm{Cl})$ & $\mathbf{P}$ & OR $(95 \% \mathrm{Cl})$ & $\mathbf{P}$ & OR $(95 \% \mathrm{Cl})$ & $\mathbf{P}$ \\
\hline $\begin{array}{l}\text { Normotension with } \\
\text { normal HCY }\end{array}$ & $69(5.4 \%)$ & 1.00 (ref) & - & - & - & - & \\
\hline $\begin{array}{l}\text { Normotension with } \\
\text { hyperhomocysteinemia }\end{array}$ & 52 (10.8\%) & $\begin{array}{l}2.145 \\
(1.472 \text { to } 3.125)\end{array}$ & $<0.001$ & 1.00 (ref) & - & - & \\
\hline $\begin{array}{l}\text { Prehypertension with } \\
\text { normal HCY }\end{array}$ & 150 (10.4\%) & $\begin{array}{l}2.049 \\
(1.525 \text { to } 2.754)\end{array}$ & $<0.001$ & $\begin{array}{l}0.956 \\
(0.684 \text { to } 1.334)\end{array}$ & $>0.05$ & 1.00 (ref) & - \\
\hline $\begin{array}{l}\text { Prehypertension with } \\
\text { hyperhomocysteinemia }\end{array}$ & 137 (15.3\%) & $\begin{array}{l}3.199 \\
\text { (2.362 to } 4.332)\end{array}$ & $<0.001$ & $\begin{array}{l}1.492 \\
\text { (1.061 to } 2.097)\end{array}$ & 0.021 & $\begin{array}{l}1.561 \\
(1.218 \text { to } 2.001)\end{array}$ & $<0.001$ \\
\hline
\end{tabular}

The ORs and $95 \%$ Cls were calculated by binary logistic regression analysis.

analysis was applied to adjust the possible confounders. A value of $\mathrm{P}<0.05$ was considered statistically significant.

\section{RESULTS}

In 4102 health examination subjects, there were 408 $(9.9 \%)$ ones detected with increased CIMT. Clinical characteristics were shown in table 1 . All characteristics were significantly different between subjects with and without increased CIMT. Age, systolic blood pressure, diastolic blood pressure, BMI, CHO, LDL, TG, fasting blood glucose (FBG) and HCY increased in subjects with increased CIMT $(\mathrm{P}<0.05)$, whereas the levels of HDL showed the reverse trend $(\mathrm{P}<0.05)$.

The subjects were divided into four groups to investigate the association of prehypertension and hyperhomocysteinemia with increased CIMT. The increased CIMT positive rate among four groups was significantly different (table 2). There were 5.4\% (69/1218), 10.8\% (52/428), $10.4 \%(150 / 1292)$ and $15.3 \%(137 / 756)$ in the control group without prehypertension or hyperhomocysteinemia, simple hyperhomocysteinemia group, isolated prehypertension group and comorbid prehypertension and

\begin{tabular}{|c|c|c|c|}
\hline & $\beta$ & OR $^{*}(95 \% \mathrm{Cl})$ & $\mathbf{P}$ \\
\hline $\begin{array}{l}\text { Normotension with } \\
\text { normal HCY }\end{array}$ & - & 1.000 (ref) & - \\
\hline $\begin{array}{l}\text { Normotension with } \\
\text { hyperhomocysteinemia }\end{array}$ & 0.211 & $\begin{array}{l}1.235 \\
(0.815 \text { to } 1.870)\end{array}$ & 0.319 \\
\hline $\begin{array}{l}\text { Prehypertension with } \\
\text { normal HCY }\end{array}$ & 0.177 & $\begin{array}{l}1.193 \\
(0.864 \text { to } 1.647)\end{array}$ & 0.283 \\
\hline $\begin{array}{l}\text { Prehypertension with } \\
\text { hyperhomocysteinemia }\end{array}$ & 0.391 & $\begin{array}{l}1.479 \\
(1.042 \text { to } 2.099)\end{array}$ & 0.028 \\
\hline
\end{tabular}

${ }^{*}$ Adjusted for age, sex, BMI, CHO, HDL-C, LDL-C, TG, FBG and smoking.

$\mathrm{BMI}$, body mass index; $\mathrm{CHO}$, total cholesterol; FBG, fasting blood glucose; HCY, homocysteine; HDL-C, high-density lipoprotein cholesterol; LDL-C, low-density lipoprotein cholesterol; TG, triglycerides. hyperhomocysteinemia group, respectively. The patients with comorbid prehypertension and hyperhomocysteinemia had a higher risk of increased CIMT, than isolated prehypertensive ones, with an OR of 1.561 (95\% CI 1.218 to 2.001, $\mathrm{P}<0.05)$. Compared with the controls, the OR in prehypertensive patients with hyperhomocysteinemia was 3.199 (95\% CI 2.362 to $4.332, \mathrm{P}<0.05)$.

Based on these results, we further explored the potential independent risk factor of increased CIMT by multivariate logistic regression analysis (table 3). Only comorbid prehypertension and hyperhomocysteinemia was independently associated with increased CIMT (OR $1.485,95 \%$ CI 1.047 to $2.108, \mathrm{P}<0.05$ ) after adjusting for age, sex, BMI, CHO, HDL-C, LDL-C, TG, FBG and smoking; however, isolated prehypertension or hyperhomocysteinemia was not.

\section{DISCUSSION}

Our study suggested that comorbid prehypertension and hyperhomocysteinemia was an independent risk factor of subclinical atherosclerosis, but simple hyperhomocysteinemia or isolated prehypertension was not, based on the cross-sectional analysis of asymptomatic Chinese subjects. This result was consistent with the former study which demonstrated that prehypertension with hyperhomocysteinemia manifested adverse cardiometabolic risk factors, such as TG, high hs-C reactive protein and uric acid. ${ }^{21}$ Aggressive approach such as screening and early management of asymptomatic population with comorbid prehypertension and hyperhomocysteinemia may contribute to delay the progression to cardiovascular disease.

Prehypertension is associated with subclinical atherosclerosis, including increased coronary atherosclerosis, ${ }^{22}$ carotid brachial intima-media thickness. ${ }^{23}$ A Swedish study demonstrated that prehypertension was associated with thickening of the intimal layer of the radial artery. ${ }^{24}$ Manios et al found that prehypertensive patients had higher common carotid artery intima-media thickness (CCA-IMT) and left ventricular mass than their normotensive counterparts. Prehypertension status seemed to cross sectionally associated with subclinical atherosclerosis 
and target-organ damage. ${ }^{25}$ However, a recent study suggested that actual prehypertensive and normotensive subjects did not differ significantly in terms of CCA-IMT values. ${ }^{26}$ In our study, there was no significant difference in the increased CIMT positive rate between prehypertensives subjects and normotensive ones after adjustment of confounders.

HCY is an acceptable risk factor for cardiovascular disease. Several studies suggested that elevated plasma HCY levels were related to increased CIMT. ${ }^{27}{ }^{28}$ However, another study of 187 patients with cardiovascular disease in Germany did not find a positive association between HCY levels and CIMT, which was consistent with our results. ${ }^{29}$ In our study, hyperhomocysteinemia was not an independently risk factor of subclinical atherosclerosis. There was no significant difference of increased CIMT positive rate between simple hyperhomocysteinemia group and control group.

Cardiovascular diseases have a complex aetiology. Age, dyslipidaemia and diabetes are established traditional risk factors for cardiovascular diseases. These risk factors interact and make progression from subclinical atherosclerosis to cardiovascular diseases. In our study, we found that isolated prehypertension or simple hyperhomocysteinemia was not independent risk factors for subclinical atherosclerosis; comorbid prehypertension and hyperhomocysteinemia seemed to synergistically aggravate the development of subclinical atherosclerosis. That might be related to endothelium dysfunction and inflammation. It is well known that hyperhomocysteinemia plays an important role in the pathogenesis of atherosclerosis, which could induce oxidative stress, endothelium dysfunction and inflammation. ${ }^{30} \mathrm{Hu}$ et al also found that structure and function impairments of carotid artery were more serious and inflammatory factors were significantly higher in the patients with H-type hypertension (comorbid hypertension and hyperhomocysteinemia) than non-H-type hypertensive ones. ${ }^{31}$ However, the clear mechanisms need further study. Early identification of apparently healthy individuals with comorbid prehypertension and hyperhomocysteinemia and control of related factors may help delay the development of subclinical atherosclerosis to clinical atherosclerosis and cardiovascular events.

There are some limitations to our study. Some definitions (hyperhomocysteinemia and increased CIMT) were according to Chinese guidelines which might be different among institutions and countries. Other confounders, such as former smoker, which we did not take them into consideration, might be a limitation. Because of the cross-sectional study design, the speculation regarding causality is limited and requires further research.

\section{CONCLUSION}

Our study suggested that comorbid prehypertension and hyperhomocysteinemia was an independent risk factor of subclinical atherosclerosis in asymptomatic Chinese, but isolated prehypertension or hyperhomocysteinemia was not. Therefore, combined intervention for prehypertension and hyperhomocysteinemia may contribute to decrease the incident of cardiovascular disease.

Contributors BL participated in the data collection and drafted the manuscript. XD participated in the data collection. GQ and ZC participated in the design of the study and undertook statistical analyses. All authors were involved in writing the paper and had final approval of the submitted and published versions.

Funding This research received no specific grant from any funding agency in the public, commercial or not-for-profit sectors.

Competing interests None declared.

Patient consent Obtained.

Ethics approval This study was reviewed and approved by the Institutional Review Board of the Second Affiliated Hospital of the Zhejiang University School of Medicine (ethical review code: Research 2014-113).

Provenance and peer review Not commissioned; externally peer reviewed.

Data sharing statement No additional data are available.

Open Access This is an Open Access article distributed in accordance with the Creative Commons Attribution Non Commercial (CC BY-NC 4.0) license, which permits others to distribute, remix, adapt, build upon this work non-commercially, and license their derivative works on different terms, provided the original work is properly cited and the use is non-commercial. See: http://creativecommons.org/ licenses/by-nc/4.0/

(c) Article author(s) (or their employer(s) unless otherwise stated in the text of the article) 2018. All rights reserved. No commercial use is permitted unless otherwise expressly granted.

\section{REFERENCES}

1. Sirimarco G, Amarenco P, Labreuche J, et al. Carotid atherosclerosis and risk of subsequent coronary event in outpatients with atherothrombosis. Stroke 2013;44:373-9.

2. Hamed SA. Atherosclerosis in epilepsy: its causes and implications. Epilepsy Behav 2014;41:290-6.

3. Herrington W, Lacey B, Sherliker P, et al. Epidemiology of Atherosclerosis and the Potential to Reduce the Global Burden of Atherothrombotic Disease. Circ Res 2016;118:535-46.

4. Lorenz MW, Markus HS, Bots ML, et al. Prediction of clinical cardiovascular events with carotid intima-media thickness: a systematic review and meta-analysis. Circulation 2007;115:459-67.

5. de Bree A, Mennen LI, Bertrais S, et al. Relation between homocysteine concentrations and structural and functional arterial parameters in a French general population. J Inherit Metab Dis 2003;26(Suppl 1):24.

6. Durga J, Bots ML, Schouten EG, et al. Homocysteine not associated with intima-media thickness. J Inherit Metab Dis 2003;26(Suppl 1):25.

7. Vermeer SE, van Dijk EJ, Koudstaal PJ, et al. Homocysteine, silent brain infarcts, and white matter lesions: the Rotterdam Scan Study. Ann Neurol 2002;51:285-9.

8. Adachi H, Hirai Y, Fujiura Y, et al. Plasma homocysteine levels and atherosclerosis in Japan: epidemiological study by use of carotid ultrasonography. Stroke 2002;33:2177-81.

9. Catena C, Colussi G, Url-Michitsch M, et al. Subclinical carotid artery disease and plasma homocysteine levels in patients with hypertension. J Am Soc Hypertens 2015;9:167-75.

10. Bogdanski P, Miller-Kasprzak E, Pupek-Musialik D, et al. Plasma total homocysteine is a determinant of carotid intima-media thickness and circulating endothelial progenitor cells in patients with newly diagnosed hypertension. Clin Chem Lab Med 2012;50:1107-13.

11. Okura T, Miyoshi $\mathrm{K}$, Irita $\mathrm{J}$, et al. Hyperhomocysteinemia is one of the risk factors associated with cerebrovascular stiffness in hypertensive patients, especially elderly males. Sci Rep 2014;4:5663.

12. Zhang $Z$, Fang $X$, Hua $Y$, et al. Combined Effect of Hyperhomocysteinemia and Hypertension on the Presence of Early Carotid Artery Atherosclerosis. J Stroke Cerebrovasc Dis 2016;25:1254-62.

13. Chen $Z$, Wang F, Zheng $Y$, et al. H-type hypertension is an important risk factor of carotid atherosclerotic plaques. Clin Exp Hypertens 2016;38:424-8. 
14. Yang G, Ma Y, Wang S, et al. Prevalence and Correlates of Prehypertension and Hypertension among Adults in Northeastern China: A Cross-Sectional Study. Int J Environ Res Public Health 2015;13:82.

15. Gonzales K, Roeber J, Kanny D, et al. Alcohol-attributable deaths and years of potential life lost--11 States, 2006-2010. MMWR Morb Mortal Wkly Rep 2014;63:213-6.

16. Liu LS. Writing Group of 2010 Chinese Guidelines for the Management of Hypertension. [2010 Chinese guidelines for the management of hypertension]. Zhonghua Xin Xue Guan Bing Za Zhi 2011;39:579-615.

17. Alkerwi A, Baydarlioglu B, Sauvageot N, et al. Smoking status is inversely associated with overall diet quality: Findings from the ORISCAV-LUX study. Clin Nutr 2017;36:1275-82.

18. Chobanian AV, Bakris GL, Black HR, et al. The Seventh Report of the Joint National Committee on Prevention, Detection, Evaluation, and Treatment of High Blood Pressure: the JNC 7 report. JAMA 2003;289:2560-72.

19. Johnsen SH, Mathiesen EB, Joakimsen O, et al. Carotid atherosclerosis is a stronger predictor of myocardial infarction in women than in men: a 6-year follow-up study of 6226 persons: the Tromsø Study. Stroke 2007;38:2873-80.

20. Chen BL, Yang Y, Yong Q, et al. Reproducibility of ultrasonic measurement of carotid artery intima-media thickness. Chinese Journal of Ultrasonography 2010;19:120-3.

21. Chen $\mathrm{H}$, Sun $\mathrm{Y}$, Wang X, et al. Association of cardiometabolic risk profile with prehypertension accompany hyperhomocysteinaemia. Clin Exp Hypertens 2015;37:218-22.
22. Washio M, Tokunaga S, Yoshimasu K, et al. Role of prehypertension in the development of coronary atherosclerosis in Japan. J Epidemiol 2004;14:57-62.

23. Toikka JO, Laine $\mathrm{H}$, Ahotupa $\mathrm{M}$, et al. Increased arterial intima-media thickness and in vivo LDL oxidation in young men with borderline hypertension. Hypertension 2000;36:929-33.

24. Myredal A, Gan LM, Osika W, et al. Increased intima thickness of the radial artery in individuals with prehypertension and hypertension. Atherosclerosis 2010;209:147-51.

25. Manios E, Tsivgoulis G, Koroboki E, et al. Impact of prehypertension on common carotid artery intima-media thickness and left ventricular mass. Stroke 2009;40:1515-8

26. Manios E, Michas F, Tsivgoulis G, et al. Impact of prehypertension on carotid artery intima-media thickening: actual or masked? Atherosclerosis 2011;214:215-9.

27. Boers GH. Hyperhomocysteinemia as a risk factor for arterial and venous disease. A review of evidence and relevance. Thromb Haemost 1997;78:520-2.

28. Bostom AG, Selhub J. Homocysteine and arteriosclerosis: subclinical and clinical disease associations. Circulation 1999;99:2361-3.

29. Linnebank M, Moskau S, Farmand S, et al. Homocysteine and carotid intima-media thickness in a german population: lack of clinical relevance. Stroke 2006;37:2840-2.

30. Zhao J, Chen H, Liu N, et al. Role of Hyperhomocysteinemia and Hyperuricemia in Pathogenesis of Atherosclerosis. J Stroke Cerebrovasc Dis 2017;26:2695-9.

31. Hu Z, Hou QZ, Zhao S, et al. [Structural and functional changes of the carotid artery and their relationship with subclinical inflammation in patients with $\mathrm{H}$-type hypertension]. Nan Fang Yi Ke Da Xue Xue Bao 2012;32:1175-8. 